D.T. Wickramasinghe, L. Ferrario, and G.V. Bicknell, eds.

\title{
A Model for WZ Sge with "Standard" Values of $\alpha$
}

\author{
J.M. Hameury \\ URA 2180 du CNRS, Observatoire de Strasbourg, 11 rue de \\ l'Université, F-6rooo Strasbourg, France
}

J.M. Huré

URA 173 du CNRS, DAEC, Observatoire de Paris, Section de Meudon, F-92195 Meudon Cedex, France

\section{J.P. Lasota}

UPR 176 du CNRS, DARC, Observatoire de Paris, Section de Meudon, F-92195 Meudon Cedex, France

\begin{abstract}
We present a model for the dwarf nova WZ Sge which does not imply an unusually - and unexplained - low value of the viscosity during low states. We propose that the inner parts of the accretion disc are disrupted by either a magnetic field or evaporation, so that the disc is stable (or very close to being stable) in quiescence, as the mass transfer rate is very low, and the disc can sit on the cool, lower branch of the thermal equilibrium curve. Outbursts are triggered by an increase of mass transfer, causing a standard disc instability, and are strongly enhanced by illumination effects. Both the recurrence time, and the characteristics of the light curve in outburst can be accounted for.
\end{abstract}

\section{Introduction}

Dwarf novae (DN) are cataclysmic variables, which, at usually irregular intervals, show a sudden increase of brightness $(2-7 \mathrm{mag})$. This eruption is now well understood as resulting from a thermal and viscous instability of the accretion disc (see Osaki, this volume, for a review); as a consequence, dwarf novae are most useful in giving us a handle on the value of the viscosity in accretion disc. Current models of dwarf novae imply values of the $\alpha$ parameter of the ShakuraSunyaev prescription for the viscosity of the order of a few percent in the low, quiescent state, and about 4-10 times larger during outbursts. Most dwarf novae fit quite well this description; the compilation of $22 \mathrm{DN}$ by Livio \& Spruit (1991) shows values of $\alpha$ in the range $0.02-0.06$ in the low state. There is however an exception to this picture: WZ Sge. WZ Sge is an extreme dwarf nova that exhibits large amplitude outbursts at very long intervals; standard DN models require values of $\alpha$ as low as $10^{-4}$ during quiescence (Smak 1993). There is however no obvious reason why the viscosity should be that low in WZ Sge, which is not grossly different from other systems. We show here that the standard disc model can be adapted, so as to account for the observational 
characteristics of WZ Sge, without requiring an anomalously low value of the viscosity.

\section{WZ Sge in quiescence}

The orbital period of WZ Sge is $81 \mathrm{~min}$, close to the minimum; the system is thus very compact, and the accretion disc is quite small, with an outer radius of $1.110^{10} \mathrm{~cm}$ during quiescence (Smak 1993), for primary and secondary masses of 0.45 and $0.06 \mathrm{M}_{\odot}$ respectively. From the luminosity of the hot spot, Smak (1993) determined a mass transfer rate $\dot{M}_{\mathrm{tr}}$ of $210^{15} \mathrm{~g} \mathrm{~s}^{-1}$.

WZ Sge has been detected by Einstein (Eracleous et al. 1991), Exosat (Mukai \& Shiokawa 1993) and ROSAT (van Teeseling et al. 1996); its X-ray luminosity did not significantly change from 4 months to more than 7 years after the 1978 outburst, and stayed at the level of $310^{30} \mathrm{erg} \mathrm{s}^{-1}$. If interpreted in terms of accretion rate onto the white dwarf surface, this would imply that the disc is not very far from steady state, and that $\alpha$ cannot be very small. Moreover, this luminosity corresponds to an accretion rate $\dot{M}_{\text {acc }}$ onto the white dwarf surface of $1.010^{14} \eta^{-1} \mathrm{~g} \mathrm{~s}^{-1}$ (excluding the contribution of the boundary layer), where $\eta$ is the efficiency of accretion in the X-ray domain. $\dot{M}_{\text {acc }}$ has to be low enough so that the inner regions of the disc are on the cool, stable branch of the $\Sigma-T_{\text {eff }}$ curve. This implies:

$$
\dot{M}<\dot{M}_{\mathrm{B}}=1.43 \times 10^{13} M_{1}^{-0.87} r_{\mathrm{g}}^{2.60} \mathrm{gs}^{-1}
$$

where $M_{1}$ is the primary mass and $r_{9}$ the radius in units of $10^{9} \mathrm{~cm}$ (Ludwig et al. 1994). This requires the radius of the inner edge of the disc $r_{\text {in }}$ to be larger than $1.6210^{9} \eta^{-0.38} \mathrm{~cm}$, assuming $M_{1}=0.45$. This is larger than the white dwarf radius, which is easily interpreted if the inner parts of the disc are disrupted by either a magnetic field (see e.g. Livio \& Pringle 1992), or by evaporation (Meyer \& Meyer-Hofmeister 1994). In the following, we assume $r_{\text {in }}=4 \times 10^{9} \mathrm{~cm}$, which ensures that the total disc luminosity is less than the luminosity of the hot spot, in agreement with observations.

\section{The outburst}

Two possible mechanisms have been proposed for triggering outbursts. The disc can be stable on the cool branch in quiescence, in which case outbursts have to be triggered by an increase of the mass transfer from the donor star, causing the disc to become thermally unstable (Lasota et al. 1996), or the disc can be marginally unstable (Warner et al. 1996). It must however be noted that, because the disc must remain on the cool, stable branch during quiescence, its mass cannot be more than the integral of the maximum $\Sigma$, i.e. $M_{\mathrm{d}}<M_{\max }=6 \times 10^{21} \alpha^{-0.8} \mathrm{~g}$ (Smak 1993). The recurrence time is the time it takes to increase $M_{\mathrm{d}}$ to this maximum value, i.e.

$$
t_{\mathrm{rec}} \sim \frac{M_{\mathrm{max}}}{\dot{M}_{\mathrm{tr}}-\dot{M}_{\mathrm{acc}}} \sim 4\left(\frac{\alpha}{10^{-2}}\right)^{-0.8}\left(1-\frac{\dot{M}_{\mathrm{acc}}}{\dot{M}_{\mathrm{tr}}}\right)^{-1} \mathrm{yr}
$$




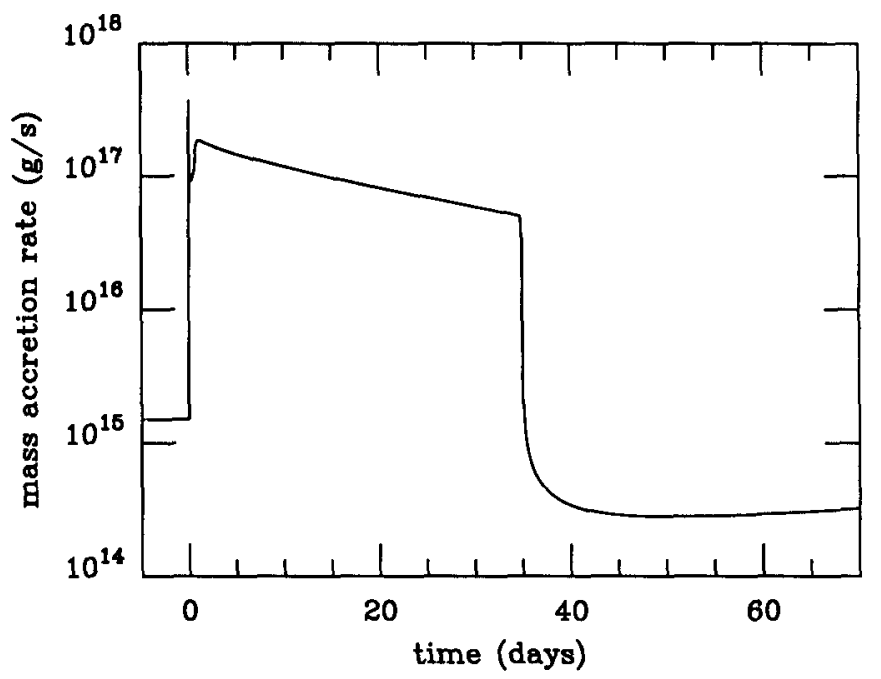

Figure 1. Predicted accretion rate for WZ Sge

If one requires that $\alpha$ is not extremely small, $\dot{M}_{\text {acc }}$ must be very close to $\dot{M}_{\mathrm{tr}}$ (within 10\%) during the whole recurrence time; it is in fact most likely that an outburst would be triggered by a small fluctuation of $\dot{M}_{\mathrm{tr}}$. Therefore, both models are in fact identical.

In the standard disc instability model, the duration of the outburst is at most the time it takes to empty the disc, i.e.

$$
t_{\text {outburst }}=\frac{M_{\max }}{\dot{M}_{\mathrm{acc}}-\dot{M}_{\mathrm{tr}}} \sim 3\left(\frac{\alpha}{10^{-2}}\right)^{-0.8}\left(1-\frac{\dot{M}_{\mathrm{tr}}}{\dot{M}_{\mathrm{acc}}}\right)^{-1} \text { days }
$$

The long duration of WZ Sge outbursts (about one month) implies, if $\alpha$ is not very small, that the mass transfer rate increases by two orders of magnitude, and is of the same order as the accretion rate during outburst. This would very naturally result from the illumination of the secondary; an increase of $\dot{M}_{\mathrm{tr}}$ by factors $\sim 2$ has been observed in normal DN such as Z Cha and U Gem (Smak 1995); illumination effects are expected (and are observed, Smak 1993) to be even more important in WZ Sge, in which the quiescent $\dot{M}_{\mathrm{tr}}$ is particularly low, and which has the shortest orbital period. The response of the secondary to illumination has been discussed by e.g. Osaki (1985) and Hameury et al. (1986), but, because of screening effects, flows from the secondary poles to $L_{1}$, and influence of the emitted spectrum, are very complex. As a preliminary step, we assume here that $\dot{M}_{\mathrm{tr}}=\gamma \dot{M}_{\mathrm{acc}}$, with $\gamma<1$; this is similar to the approach of Augusteijn et al. (1993) in the context of X-ray novae.

The expected outcome of the model is that, once the heat wave reaches the inner edge of the disc, $\dot{M}_{\text {tr }}$ increases up to a value $\gamma \dot{M}_{\text {acc }}$, and then decays following an exponential law with a decay time equal to $t_{\text {visc }} / \ln \gamma$, where $t_{\text {visc }}$ is the viscous time of the disc. When $\dot{M}_{\text {tr }}$ becomes less than the critical value 
below which the hot, stable solution in the $\Sigma-T_{\text {eff }}$ diagram does not exist any longer, a cooling wave starts from the outer edge of the disc, and brings it into quiescence.

In order to test this, we have calculated the time-dependent evolution of a disc initially stable with a low $\dot{M}_{\mathrm{tr}}$, taken to be $1.5 \times 10^{15} \mathrm{~g} \mathrm{~s}^{-1}$, which is suddenly increased to $\max \left(5 \times 10^{15}, 0.87 \dot{M}_{\text {acc }}\right) \mathrm{g} \mathrm{s}^{-1} . \alpha$ is taken to be 0.01 in the cool branch and 0.1 in the hot one. All other parameters are those quoted here for WZ Sge; the code used is described in Hameury et al. (1996). The results are displayed in Fig. 1. It is seen that the shape of the light curve is in good agreement with observations.

\section{Conclusion}

We have shown that the unusually long recurrence time and outburst duration in WZ Sge does not require the viscosity in this system to be much lower than in all other systems; these characteristics would result from (1) a low value of the mass transfer rate; (2) a truncated disc, which is required in many other systems to account for e.g. the observed optical-UV delay; and (3) a significant illumination effect. WZ Sge would thus be explained by a combination of the two different model proposed by Osaki $(1974,1985)$, in a way similar to the proposition of Duschl \& Livio (1989).

\section{References}

Augusteijn, T., Kuulkers, E., \& Shaham, J. 1993, A\&A, 279, L9

Duschl, W. J., \& Livio, M. 1989, A\&A, 209, 183

Eracleous, M., Halpern, J., \& Patterson, J. 1991, ApJ, 290,300

Hameury, J. M.., King, A. R., \& Lasota, J. P. 1986, A\&A, 162, 71

Hameury, J. M., Huré, J. M., \& Lasota, J. P., in preparation

Lasota, J. P., Hameury, J. M., \& Huré, J. M. 1996, A\&A, 302, L29

Livio, M., \& Pringle, J. 1992, MNRAS, 259, 23p

Livio, M., \& Spruit, H.C. 1991, A\&A, 252,189

Ludwig, K., Meyer-Hofmeister, E., \& Ritter, H. 1993, A\&A, 290, 473

Meyer, F., \& Meyer-Hofmeister, E. 1994, A\&A, 288, 175

Mukai K., \& Shiokawa, K. 1993, ApJ, 418, 863

Osaki, Y. 1974, PASJ, 26, 429

Osaki, Y. 1985, A\&A, 144, 369

Osaki, Y. 1995, PASJ, 47, 47

Smak, J. 1993, Acta astron., 43, 101

Smak, J. 1995, Acta astron., 45, 355

van Teeseling, A., Beuermann, K., \& Verbunt, F. 1996, A\&A, in press

Warner, B., Livio, M., \& Tout, C. A. 1996, MNRAS, in press 